\title{
Combining Energy and Power based Safety Metrics in Controller Design for Domestic Robots
}

\author{
Tadele Shiferaw Tadele, Theo J.A. de Vries, and Stefano Stramigioli
}

\begin{abstract}
This paper presents a general passivity based interaction controller design approach that utilizes a combined energy and power based safety norms to assert safety of domestic robots. Since these robots are expected to co-habit the same environment with a human user, analysing and ensuring their safety is an important requirement. Safety analysis of domestic robots determine whether a robot achieves a desired safety level according to some quantitative safety metrics. When it comes to controller design for human friendly robots, it often involves introducing compliance and ensuring asymptotic stability using impedance control technique and passivity theories. The controller proposed in this work also uses a passive design that extends the standard impedance control scheme with energy and power based safety metrics to ensure that safety requirements defined in these norms are achieved by domestic robots. The effectiveness of the proposed guideline is illustrated with simulation and experimental results.

Index Terms-Domestic Robots, Impedance Control, Safety Metric, Passivity Based Control, PD, Interaction Control
\end{abstract}

\section{INTRODUCTION}

Recent advances in the robotics field have accelerated the development of domestic robots that can operate as assistants in home or office activities. Safety of these robots is of paramount importance because the robots operate in a human present environment. A standard safety analysis in robotics is done by using a risk based method that involves the following three steps: (1) risk assessment; (2) risk elimination and reduction; (3) validation and verification [1]. The first two steps are used to identify risks, define possible injuries and suggest design solutions that can minimize or if possible eliminate the risks. The final validation step defines qualitative or quantitative safety norms that can be used to evaluate safety of the robotic system.

These norms consist of safety criteria that should be met to achieve safer systems as well as quantitative safety metrics that are used evaluate results of a safety modification and assist accreditation procedures. Domestic robots also require meaningful norms to evaluate their safety before they can be successfully deployed around human users. For a complex system like a domestic robot, there are a number of risks and injuries that demand several safety metrics, each dealing with a specific risk. In this publication, we will only consider collision risks together with the injuries and safety metrics related with them. There are different safety

This work was supported by funds from the Netherlands Ministry of Economic Affairs under research project Bobbie.

The authors are with the Robotics and Mechatronics chair at the Faculty of Electrical Engineering, Mathematics and Computer Science, University of Twente, 7500 AE Enschede, The Netherlands. E-mail: \{t.s.tadele, t.j.a.deVries, s.stramigioli\}@utwente.nl metrics that deal with collision injuries and they consider acceleration, force, energy or power as a main parameter for the safety analysis. Though these metrics are essential in identifying and analysing possible injuries, their exploitation in controller design has been minimal in literature. Control schemes that incorporate safety norms in their design identify an appropriate metric and propose solutions that guarantee safety either by using a pure control action or modifying the reference trajectory input to the controller [2], [3], [4].

Domestic robots are designed to operate in an unstructured environment and also to interact with unknown objects. Thus, the controller design in domestic robots should incorporate safety issues such as ensuring asymptotic stability even during interaction, introducing compliance to minimize injury in case of uncontrolled impact and providing robustness to parametric and modeling uncertainty. Different authors have exploited passivity theories in the control law design to guarantee asymptotically stability of domestic robots [5], [6]. Passive systems are stable dynamic systems whose bounded total energy can be used to prove Lyapunov stability even during interaction with unknown passive environment[7]. The safety demand for a compliant robotic manipulator is achieved using an impedance control technique which allows modification of the dynamic characteristic of a manipulator to achieve a desirable compliant behaviour [8]. Impedance control is an interaction control scheme in which an energetic bidirectional signal exchange between the controller and the plant results in a desired dynamic behavior of the system [9].

With a general goal of detecting and avoiding unsafe conditions via control action, this work presents a novel control scheme that incorporates safety metrics in impedance control design. The control scheme begins with a basic impedance controller tuned according to a certain performance requirement [10], [11] and then modifies the controller parameters so that safety limits defined in a combined energy and power based metrics are met. This variable impedance controller is realized using an energy tank based implementation to guarantee the passivity and energy consistency of the system.

This paper is organized as follows. In section II safety norms used to address collision risks in domestic robots are briefly presented and previous works regarding controller design using these safety norms are reviewed. Then section III discusses details of the proposed combined safety norm and controller design. Section IV presents simulation and experimental results before section $\mathrm{V}$ summarizes the main ideas and presents the conclusions. 


\section{BACKGROUND}

\section{A. Safety Metrics in Domestic Robotics}

The most common safety metric in domestic robotics is the Head Injury Criteria (HIC) which is also used in biomechanics study and accident researches. Human biomechanics data defined in the Wayne State Concussion Tolerance Curve is the basis of this safety metric and it is computed by using measured acceleration of a human head due to an impact [12]. Mathematically it is given as,

$$
H I C_{\Delta t}=\Delta t\left[\frac{1}{\Delta t} \int_{0}^{\Delta t} a(\tau) d \tau\right]^{2.5}
$$

where $a(\tau)$ is the head acceleration measured in function of $g$ and $\Delta t$ is measurement duration which is often taken as $15 \mathrm{~ms}$ to investigate head concussions. Though HIC is a standard metric in the automotive industry and is also used in the robotics domain, different authors have suggested that it is not an adequate metric in domestic robotics because of differences in operating velocity and injury types between the automotive and robotics domain [13], [14]. It was also reported that due to the low operating velocity of robots, even the heaviest of robots are deemed safe for humans according to HIC measurements [15].

In addition to HIC, other approximations were proposed to fit the Wayne State Tolerance data and one of them suggests reducing the power in equation (1) from 2.5 to 2 [16]. Accordingly, the final equation becomes

$$
\begin{gathered}
f=\Delta t \cdot a_{a v e}{ }^{2} \\
f=\frac{\Delta V^{2}}{\Delta t}
\end{gathered}
$$

where $a_{a v e}$ is the average acceleration and $\Delta V$ is the change in velocity of the head.

From this physically interpretable equation, possible injury to a human head is identified to be proportional to the rate of kinetic energy transferred during impact. This rate of energy transfer was previously suggested as an injury measure of constrained organs in the viscous criterion safety metric [17]. The viscous criterion proposes that injury to human organs is proportional to the product of the compression and the rate of compression. Based on the observation from equation (3), a power based safety metric known as Head Impact Power (HIP) was proposed to analyze head injuries [16]. The metric uses the probability of a concussion injury from an impact on a human head to identify the minimum power that can cause injury to a human head. According to the experiments, the power limits identified are

$$
P_{\text {limit }}= \begin{cases}12 K W & \text { for frontal impacts } \\ 10 K W & \text { for non-frontal impacts }\end{cases}
$$

Another class of safety norms used in domestic robots considers excessive force as the cause of injuries and use the maximum allowed force that can be exerted by the robot as an appropriate safety norm in domestic robots. Since the allowed tolerance limits for different body parts are not the same, it was suggested that the smallest force tolerance, which is the tolerance to neck injuries, be chosen as an operating limit [18]. In addition to the maximum limits, other force based metrics were also introduced to address safety of domestic robots. A unitless danger index defined as the ratio of force exerted by a manipulator to the maximum allowed force limit was suggested as a safety metric for mechanical and control system design of manipulators [19]. The maximum impact force that can be exerted by a multi DOF manipulator was used to define another force based safety metric called impact potential $\pi$ in [2]. The impact potential is defined mathematically as,

$$
\pi=\sup _{p \in P} \pi_{p}
$$

where $\pi_{p}$ is worst case impact forces at contact point $p$ on the surface of the robot and $P$ is the set of all possible surface contact points.

Energy based injury analysis that could be used in robotics were obtained from two experimental investigations that identified the minimum amount of energy that is required to cause failure of cranial bones and fracture of the neck bones in [20] and [21] respectively. The energy limit on cranial bone failure can be used to analyse safety during collision with a clamped human user while unclamped collisions, where the neck is free to move and bend after a collision on the head, could be evaluated based on neck fracturing energy limit. These results can then be combined to give a complete energy based safety norm for domestic robots in both cases of clamped or unclamped impacts.

$$
E_{\text {limit }}= \begin{cases}517 J & \text { adult cranium bone failure } \\ 127 J & \text { infant cranium bone failure } \\ 30 J & \text { neck fracture }\end{cases}
$$

\section{B. Safety Metrics in Controller Design}

While addressing the issue of human friendly robots that should meet a defined safety and performance requirements, [3] used HIC as a safety criteria to identify a performance limit given in terms of maximum allowed link velocity. Hence one control objective is to guarantee that the desired trajectories of the manipulator confers to the safety imposed velocity limitation. The achievable trade-off between safety and performance was evaluated for different actuation mechanisms and possible controller schemes for variable stiffness actuated manipulators were suggested.

After introducing impact potential $\pi$ as a safety norm, authors in [2] proposedan Impact Potential Controller design which ensures that impact potential of a manipulator is within a safe limit. The proposed controller possesses a hierarchical implementation with low level motion controller and higher level safety protection layer. The motion controller generates required torque output to achieve a desired motion trajectory and the safety layer checks the impact potential that will be achieved due to the motion controller torque. If the impact potential is within the safe limit it is passed onto the manipulator and if not the torque is clipped to an appropriate value to avoid possible unsafe collisions. 
[4] used energy based safety norms discussed in the previous subsection II-A to design an energy regulation controller that limits the total energy of a manipulator within the required safety limit. Taking a flexible manipulator driven by a series elastic actuator, the controller monitors the kinetic and potential energy of the manipulator and attempts to limit the overall energy of the system remains within the acceptable level. An inner position controller drives the manipulator while the energy regulation controller monitors energy content and modulates the desired trajectory reference to achieve acceptable energy level.

\section{Proposed Controller Design}

Noting the presence of a direct interaction between a controller and a plant, analysing controllers as dynamic systems that can influence the plant to achieve a desired system behaviour expands the controller design technique beyond the classical signal based design [22]. This control design approach grants an insightful view of the system, simplifies tuning and allows an overall stability analysis by using the total energy as a Lyapunov function. Consider a simplified 1-DOF robotic manipulator modeled as a mass $m$ at position $x$ and it is to be moved to a desired position $x_{d}$, a simple physical controller that can achieve the required behaviour consists of a spring connected between the desired virtual point and the mass, See Fig 1. A damper is then added to achieve an asymptotically stable system. Finally the overall system behaves like a mass-spring-damper whose behaviour can be shaped by adjusting the controller parameters.

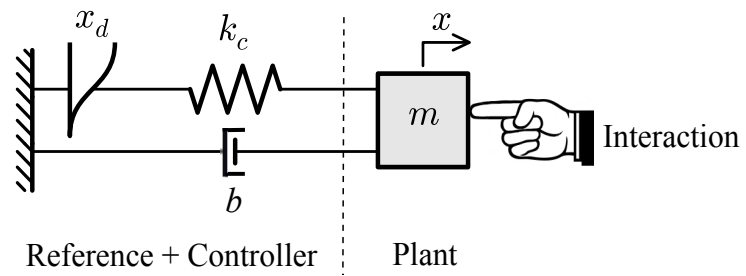

Fig. 1. Impedance controlled system.

The resulting control system is a conventional PD controller that guarantees closed loop stability for any positive gains, with the proportional term representing the spring and the derivative term representing the damper. In addition to placing the object at the desired position in a stable way, a desired system compliance and dynamic behaviour that meets performance requirements can be set by choosing an appropriate value for the controller stiffness and damping parameters $k_{c o}$ and $b_{o}$.

In addition to its passive nature and the compliance it adds in the system, this impedance controller with unmodified driving point inertia can be more human-friendly by incorporating a safety metric in its design to minimize possible injuries. Further more, by combining safety metrics that address different injuries, the controller can be designed with increased safety coverage over multiple risks. In this paper, both energy and power based metrics were used to enhance safety against possible injuries addressed by these metrics.
The physically interpretable impedance controller design belongs to a class of energy shaping controllers whose energy content is analysed during design [22]. Given the impedance controlled system shown in Fig 1, total energy of the overall system is the sum of the kinetic energy of the plant and the potential energy of the controller spring. It is given as

$$
E_{t o t}=\frac{1}{2} k_{c} x_{e}{ }^{2}+\frac{1}{2} m \dot{x}^{2}
$$

where $x_{e}=x_{d}-x$ is the motion error which is equal to the state of the controller spring and $\dot{x}$ is velocity of the plant.

So at any instant, given measured plant position, plant velocity and desired position, the overall energy of the system can be limited to a maximum allowed limit $E_{\max }$ by adjusting the stiffness of the controller spring $k_{c}$. As a result the controller stiffness parameter can be written as,

$$
k_{c}= \begin{cases}k_{c o} & \text { if } E_{t o t} \leq E_{\max } \\ \frac{2 E_{\max }-m \dot{x}^{2}}{\left(x_{d}-x\right)^{2}} & \text { if } E_{t o t}>E_{\max }\end{cases}
$$

Once the safe energy restrictions are ensured by the stiffness choice, the maximum power that the manipulator can transfer to a human during collision can be limited via a control action since the controller is the source of the power that will be transferred to a human during contact. This power restriction can be enforced in the controller by modifying the dissipative damping parameter of the controller. That is, given the impedance characteristic of the controller which accepts velocity input and outputs a force, the power conjugate of velocity, the power flowing from the controller to the plant $P_{c}$ becomes,

$$
P_{c}=\left(k_{c}\left(x_{d}-x\right)-b \dot{x}\right) \dot{x}
$$

Then for a fixed controller stiffness $k_{c}$, the power flow between the plant and controller can be limited to maximum value $P_{c_{\max }}$ by adjusting the damping parameter $b$ as follows,

$$
b= \begin{cases}b_{o} & \text { if } P_{c} \leq P_{c_{\max }} \\ \frac{k_{c}\left(x_{d}-x\right) \dot{x}-P_{c_{\max }}}{\dot{x}^{2}} & \text { if } P_{c}>P_{c_{\max }}\end{cases}
$$

Thus, when unwanted energy is identified in the system the controller drains energy by being more compliant and when unsafe power output is detected the controller increases the damping on its output. With the appropriate parameters identified using equations (8) and (10), the impedance controller implementation can be written mathematically as,

$$
F_{c}=k_{c} \cdot\left(x_{d}-x\right)-b \cdot \dot{x}
$$

Note that for a controller that is interacting with a plant via energy exchange, direct implementation of this controller with variable stiffness and damping parameters for improved safety contradicts with energetically consistent interaction controller design. That is, under constant parameter controller implementation, its energy based interaction with the plant dictates that if the energy content of the controller decreases, the same energy flows to the plant and if the energy 
content of the controller increases then it means energy is extracted from the plant. Furthermore, the modification of the stiffness parameter allows internal production of energy in the controller and results in loss of its passivity property.

To avoid this problem we propose to implement the controller using an energy tank based approach where a controller is designed as a power flow modulator between an energy storage tank and a plant. See Fig 2. This controller is also physically interpretable and consists of an energy storage tank $C S$ which behaves like a spring with a stiffness value of 1 , a modulated transmission $M T$ and a computational unit $C U$ as shown in Fig. 2. The computational unit calculates a modulating factor $u$ which determines how power flows between the storage element $C S$ and the plant. If $u=0$ the storage tank and the plant will be isolated with no energy exchange between them. As the complete system is an interconnection of energy converting transformation element and intrinsically passive mass and spring elements, it is passive by construction. Without interactions its total energy is bounded and its stability is guaranteed as long as it is interacting with passive systems.

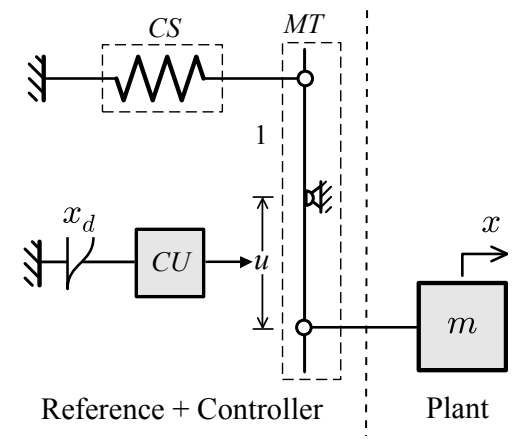

Fig. 2. Energy tank based controller design.

The port Hamiltonian equation of the tank based system shown in Fig 2 above can be written as

$$
\left(\begin{array}{c}
\dot{s} \\
\dot{p}
\end{array}\right)=\left(\begin{array}{cc}
0 & u \\
-u & 0
\end{array}\right)\left(\begin{array}{c}
s \\
p / m
\end{array}\right)
$$

,where $s$ is state of the spring like storage $C S$ which is equal to its force output, $p$ is the momentum of the plant and $p / m=\dot{x}$ is velocity of the plant.

Using the second half of equation (12), any desired impedance controller output force $F_{c}$ can be exerted on the plant by choosing the desired modulating factor $u_{d}$ as

$$
u_{d}=\frac{-F_{c}}{s}
$$

In order to ensure energetic passivity of the controller, the parameter $u$ should ensure that power flows from the storage tank $C S$ to the plant only if there is minimum available energy remaining in the tank. Thus, the modulating parameter $u$ is finally implemented as

$$
u= \begin{cases}0 & \text { if }\left((H(C S)<\epsilon) \wedge\left(P_{c}>0\right)\right) \\ \frac{-F_{c}}{s} & \text { otherwise }\end{cases}
$$

where $F_{c}$ is desired output controller torque, $H(C S)=\frac{1}{2} s^{2}$ is energy in the tank, $\epsilon$ is the minimum energy required in the tank for power to flow towards the plant and $P c=F_{c} \dot{x}$ is the power flowing from the controller to the plant.

\section{Simulation and Experimental Results}

\section{A. Simulation Results}

In order to validate the controller design method proposed in the previous section, a simulation was performed on a computer model plant with a link mass of $m=1[K g]$. To achieve a desired performance requirement and a critically damped dynamic behaviour, the impedance controller parameters were chosen as $k_{c o}=100 \mathrm{~N} / \mathrm{m}$ and $b_{o}=20 \mathrm{Ns} / \mathrm{m}$ respectively. As a proof of concept, the allowed safe energy limit is set to $E_{\max }=6 \mathrm{~J}$ and the maximum allowed power that flows from the controller is set to $P_{c_{\max }}=4 \mathrm{~W}$. Simulation results in Fig 3 and 4 show that the system was able to reach its desired position while the controller adjusted its parameters to ensure the energy of impedance controlled system as well as the power flow from the controller to the plant stayed within the allowed limits.

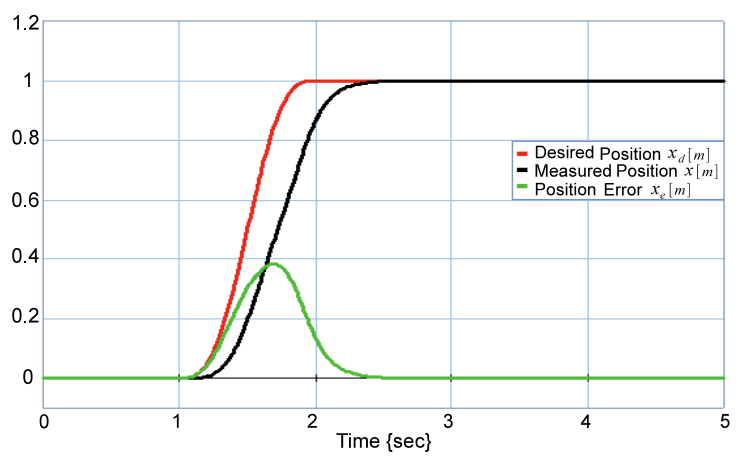

Fig. 3. Simulation results - Trajectory tracking

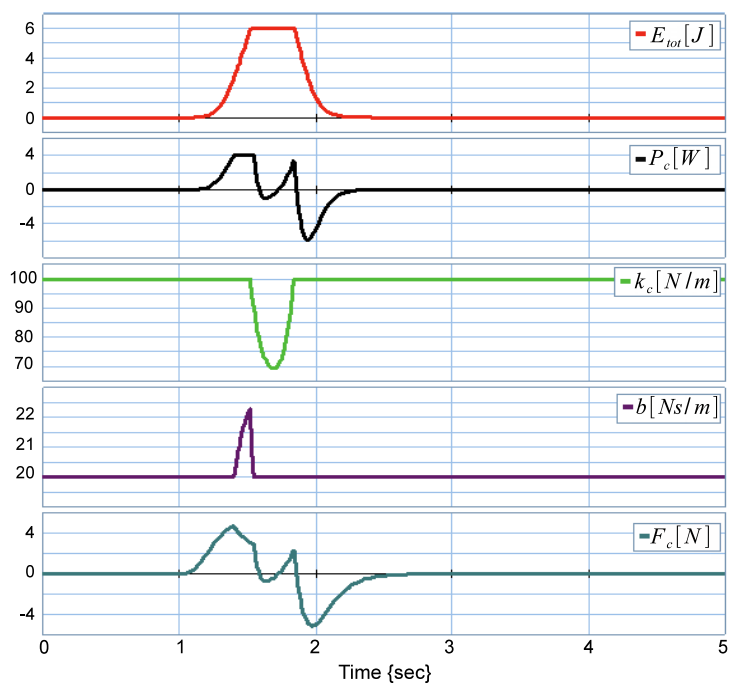

Fig. 4. Simulation results - Total energy of impedance controlled system $E_{t o t}$, power flow from the controller to the plant $P_{c}$, stiffness of the system $k_{c}$, damping ratio $b$ and output force from controller $F_{c}$ 
The controller designed resulted in an intuitive control action which can be physically interpreted. As can be seen from Fig 4, as soon as the power restriction limit is reached the controller increased its damping to oppose the spring action and lower the force transmitted from the controller. Then the energy limit was reached and the stiffness was lowered resulting in a further decrease of the controller output. At this instant the damper doesn't have a strong spring action to counter so it was lowered accordingly.

\section{B. Experimental Results}

In order to investigate practical implementation of the proposed tuning method, two experiments were performed on a 1-DOF manipulator with link inertia of $J=0.0069 \mathrm{Kgm}^{2}$ shown in Fig 5.

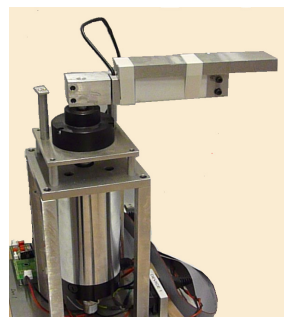

Fig. 5. A 1 D.O.F experimental setup used

On the first experiment a contact free trajectory tracking experiment was performed with maximum allowed energy limit of $E_{\max }=0.05 \mathrm{~J}$ and power limit of $P_{c_{\max }}=$ $0.1 W$ used as safety criteria limits. The initial stiffness and damping ratio of the controller were chosen, based on a performance requirement, as $k_{c}=3.5 \mathrm{Nm} / \mathrm{rad}$ and $b=0.17 \mathrm{Nms} / \mathrm{rad}$. As seen from the experimental results shown in Fig 6 and 7, the controller was able to position the plant at the desired position while keeping the safety limits by modifying its compliance and damper parameters.

In order to evaluate performance variations due to the safety criteria imposed on the system, the free motion experiment was done using a constant parameter impedance controller of $k_{c}=3.5 \mathrm{Nm} / \mathrm{rad}$ and $b=0.17 \mathrm{Nms} / \mathrm{rad}$ and its result was compared with safety limited variable impedance controller. The two controllers have similar performance under normal condition and when the safety aware controller became more compliant to keep the limits, an increase in motion tracking error was observed. See Fig 8.

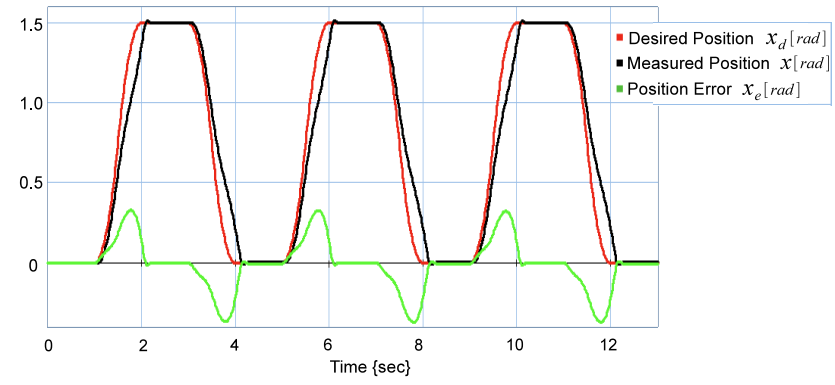

Fig. 6. Experimental results - Interaction free trajectory tracking

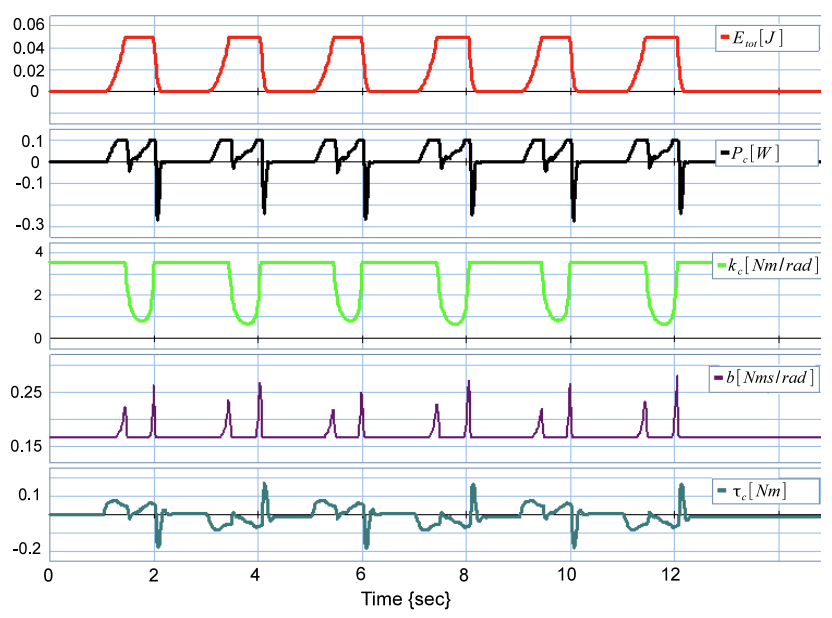

Fig. 7. Experimental results (Free motion) - Total energy of impedance controlled system $E_{t o t}$, power flow from the controller to the plant $P_{c}$, stiffness of the system $k_{c}$, damping ratio $b$ and controller torque output $\tau_{c}$

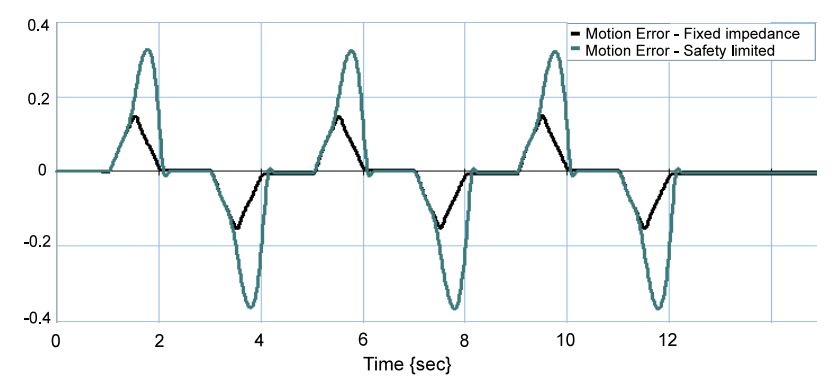

Fig. 8. Experimental results - Motion Error using an impedance controller with and without safety requirements

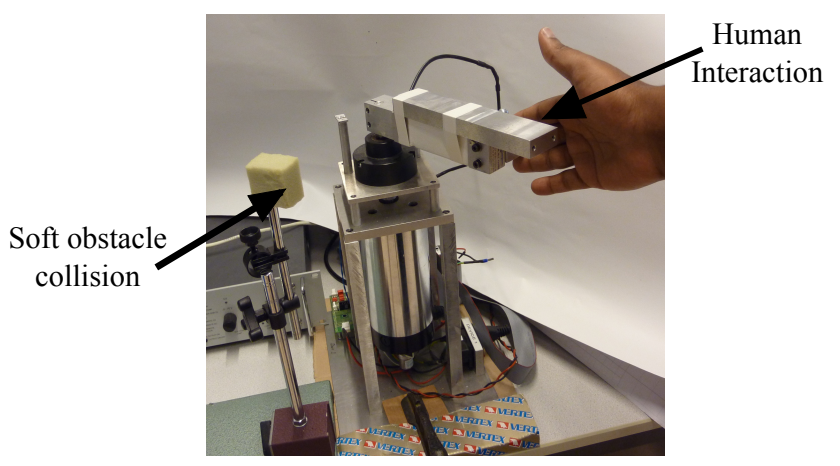

Fig. 9. Collision and interaction experiment

On the next experiment the controller action was evaluated during collision with a soft obstacle and interaction with a human user. The maximum allowed energy limit of $E_{\max }=$ $0.075 \mathrm{~J}$ and power limit of $P_{c_{\max }}=0.15 \mathrm{~W}$ were used as safety criteria limits of this experiment and the initial controller parameters were kept at $k_{c}=3.5 \mathrm{Nm} / \mathrm{rad}$ and $b=0.17 \mathrm{Nms} / \mathrm{rad}$. Experimental results shown in Fig 10 indicate that the variable impedance controller was able to increase its compliance and damping injection during collisions as well as interactions when energy and power safety limits were violated. 


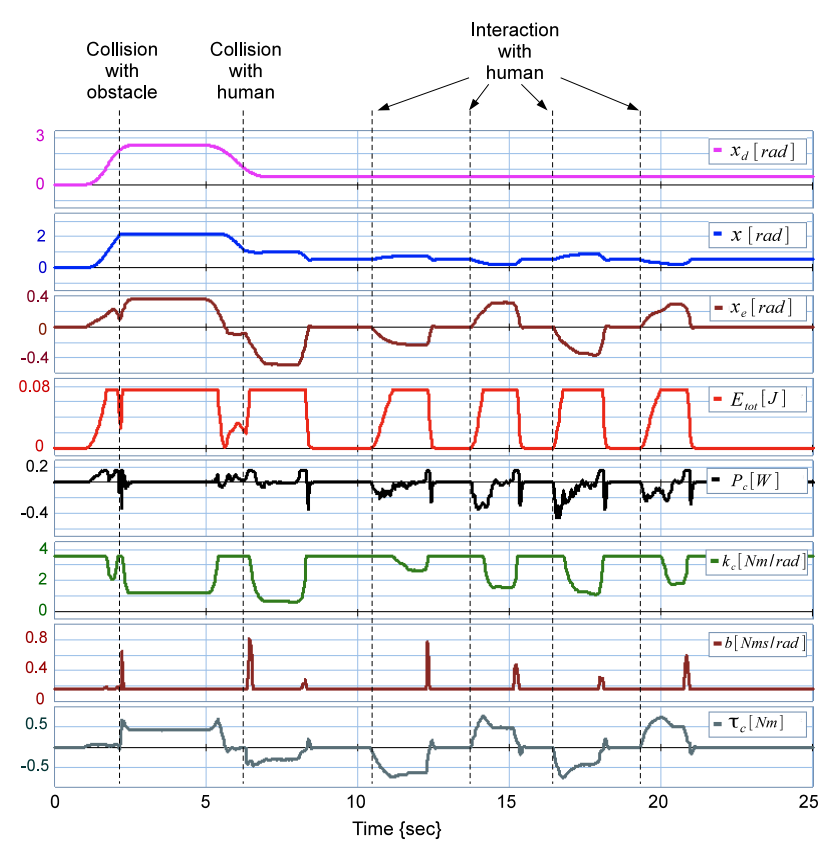

Fig. 10. Experimental results - Desired position $x_{d}$, measured position $x$, position error $x_{e}$, total energy of impedance controlled system $E_{t o t}$, power flow from the controller to the plant $P_{c}$, stiffness of the system $k_{c}$, damping ratio $b$ and controller output torque $\tau_{c}$

\section{CONCLUSION}

In this paper a controller design approach that combines impedance control, passivity based design and safety metrics was proposed for domestic robots. By analysing the energy content of a standard impedance controlled manipulator and the power flow between the plant and the controller, a combined energy and power based safety metrics were used for on-line adjustment of the controller parameters to ensure safety. To avoid the loss of passivity due to the instantaneous modulation of the controller parameters, an energy tank based implementation was used. In addition to the overall passivity achieved by the design, its flexibility also allows update of the safety limits depending on the task or the operating environment. Simulation and experimental results on a 1-DOF manipulator indicate that the controller handles both energy and power safety limits by varying its impedance. A trade-off between safety and performance was observed in an experiment where an increase in compliance resulted higher trajectory error. While the presence of friction can negatively affect system performance, its dissipative nature acts in favour of the safety requirement by decreasing the power transferred to the moving plant even below the controller limited threshold value.

Future work on this design approach will focus on safety aware impedance control of multiple D.O.F robotic manipulators. This multidimensional extension considers cartesian space impedance controllers and should deal with additional complexity due to issues such non-linearity of the plant dynamics and presence of friction. It will also investigate ways to include additional safety features such as collision detection and reaction in this controller design scheme.

\section{REFERENCES}

[1] C. Harper and G. Virk, "Towards the development of international safety standards for human robot interaction," International Journal of Social Robotics, vol. 2, pp. 229-234, 2010.

[2] J. Heinzmann and A. Zelinsky, "Quantitative Safety Guarantees for Physical Human-Robot Interaction," The International Journal of Robotics Research, vol. 22, no. 7-8, pp. 479-504, 2003.

[3] A. Bicchi and G. Tonietti, "Fast and "soft-arm" tactics [robot arm design]," Robotics Automation Magazine, IEEE, vol. 11, no. 2, pp. 22-33, 2004.

[4] M. Laffranchi, N. Tsagarakis, and D. Caldwell, "Safe human robot interaction via energy regulation control," in Proceedings of the 2009 IEEE/RSJ International Conference on Intelligent Robots and Systems, Oct 2009 , pp. 35-41.

[5] A. Albu-Schäffer, C. Ott, and G. Hirzinger, "A unified passivitybased control framework for position, torque and impedance control of flexible joint robots," The International Journal of Robotics Research, vol. 26, no. 1, pp. 23-39, 2007.

[6] A. Zanchettin, B. Lacevic, and P. Rocco, "A novel passivity-based control law for safe human-robot coexistence," in Intelligent Robots and Systems (IROS), 2012 IEEE/RSJ International Conference on, 2012, pp. 2276-2281.

[7] R. Ortega, J. A. L. Perez, P. J. Nicklasson, and H. J. Sira-Ramirez, Passivity-based Control of Euler-Lagrange Systems: Mechanical, Electrical and Electromechanical Applications, ser. Communications and Control Engineering. Springer, 2010.

[8] N. Hogan, "Impedance control - An approach to manipulation. I Theory. II - Implementation. III - Applications," ASME Transactions Journal of Dynamic Systems and Measurement Control B, vol. 107, pp. 1-24, Mar. 1985.

[9] S. Stramigioli, Modeling and IPC control of interactive mechanical systems A coordinate-free approach, ser. Lecture Notes in Control and Information Sciences. Springer, 2001.

[10] H. Kazerooni, T. Sheridan, and P. Houpt, "Robust compliant motion for manipulators, part I: The fundamental concepts of compliant motion," Robotics and Automation, IEEE Journal of, vol. 2, no. 2, pp. 83-92, 1986.

[11] T. S. Tadele, T. de Vries, and S. Stramigioli, "PID motion control tuning rules in a damping injection framework," in American Control Conference (ACC), 2013, 2013, pp. 4957-4962.

[12] J. Versace, "A review of severity index," in Proceedings of Stapp Car Crash Conference, 1971, pp. 149-170.

[13] A. D. Santis, B. Siciliano, A. D. Luca, and A. Bicchi, "An atlas of physical humanrobot interaction," Mechanism and Machine Theory, vol. 43 , no. 3 , pp. $253-270,2008$.

[14] M. V. Damme, P. Beyl, B. Vanderborght, R. V. Ham, I. Vanderniepen, A. Matthys, P. Cherelle, and D. Lefeber, "The role of compliance in robot safety," in Seventh IARP Workshop on Technical Challenges for Dependable Robots in Human Environments, 2010, pp. 65-71.

[15] S. Haddadin, A. Albu-Schffer, and G. Hirzinger, "Requirements for safe robots: Measurements, analysis and new insights," The International Journal of Robotics Research, vol. 28, no. 11-12, pp. 1507$1527,2009$.

[16] J. A. Newman, N. Shewchenko, and E. Welbourne, "A proposed new biomechanical head injury assessment function - the maximum power index," in Stapp Car Crash Conference Forty-fourth Proceedings, 2000, pp. 215-247.

[17] F. DiLorenzo, Power and Bodily Injury. Society of Automotive Engineers, 1976.

[18] J.-J. Park and J.-B. Song, "Collision analysis and evaluation of collision safety for service robots working in human environments," in International Conference on Advanced Robotics, ICAR 2009., 2009, pp. 1-6.

[19] K. Ikuta, H. Ishii, and M. Nokata, "Safety evaluation method of design and control for human-care robots," The International Journal of Robotics Research, vol. 22, no. 5, pp. 281-297, 2003.

[20] J. L. Wood, "Dynamic response of human cranial bone," Journal of Biomechanics, vol. 4, no. 1, pp. 1 - 12, 1971.

[21] N. Yoganandan, F. Pintar, D. Maiman, J. Cusick, A. S. Jr, and P. Walsh, "Human head-neck biomechanics under axial tension," Medical Engineering \& Physics, vol. 18, no. 4, pp. 289 - 294, 1996.

[22] R. Ortega, A. Van Der Schaft, I. Mareels, and B. Maschke, "Putting energy back in control," Control Systems, IEEE, vol. 21, no. 2, pp. 18-33, 2001. 